\title{
THE HOT ELECTRON EFFECT IN DOUBLE HETEROJUNCTION BIPOLAR TRANSISTORS: THEORY AND EXPERIMENT
}

\author{
Chung-Zen Chen, Si-Chen Lee and HaO-Hsiung Lin \\ Department of Electrical Engineering, National Taiwan University, Taipei, Taiwan, Republic of China
}

(Received 12 November 1987; in revised form 22 June 1988)

\begin{abstract}
A simplified analytical formulation was derived to model the hot electron effect in AlGaAs double heterojunction bipolar transistors. By fitting the common-emitter current-voltage characteristics of a real device with theory, the hot electron effect is demonstrated.
\end{abstract}

\section{INTRODUCTION}

The use of higher bandgap emitter, collector and heavily-doped base in the NPN AlGaAs double heterojunction bipolar transistor (DHBT) improves the transistor's current gain and switching speed at the same time[1-2], but results in an offset voltage related to heterojunction potential spikes[1-7]. A transport theory recently developed[8] suggests that the offset voltage problem can be suppressed by just using equal emitter-base $(e-b)$ and base-collector $(b-c)$ junction areas. Then, the $e-b$ junction potential spike $\left(\Delta E_{1}\right)$ can be preserved to provide a launching $\operatorname{pad}[9,10]$ for injecting hot electrons which further improves the frequency response of the transistor. This hot electron effect has been modeled using Monte Carlo method $[11,12]$ and experimentally studied using hot electron spectroscopy[13]. It is believed, however, to fully explore the hot electron effect, an analytical theory with minimum number of adjustable parameters is needed. In this paper, we present a simplified analytical model which is basically an extension of the previous transport theory[8] to treat the hot electron effect. Experimentally, the hot electron effect can be studied using a DHBT with both $e-b$ and $b-c$ junction potential spikes $\left(\Delta E_{2}\right)$ present[13]. The existence of $\Delta E_{2}$ causes a serious voltage dependent collector current (reach-through effect) in the common-emitter current-voltage $(I-V)$ characteristics which is very sensitive to the device parameters[14]. Therefore, the hot electron effect can be probed by fitting the expcrimental $I-V$ curve with the analytical model using independently measured device parameters.

\section{THEORETICAL MODEL}

The model is following: the injected nonequilibrium electrons across the $e-b$ heterojunction are treated by random walk process[15] when they pass through the base layer for the first time. Various scattering mechanisms such as impurity, acoustical, optical phonon, pizoelectric, hole plasmon scatterings will reduce the electron's energy and deflect its trajectory according to the energy and momentum conservation laws. Due to the high kinetic energy of the injected electrons, the optical phonon scattering and hole plasmon excitation[16] are considered to be the dominant scattering mechanisms. The "hot electrons" are defined to be those part of electrons that have higher kinetic energy than $\Delta E_{2}$ when they reach the $b-c$ junction potential spike in the first passage of the base layer as shown in Fig. 1. The probability of an electron absorbing or emitting an optical phonon is proportional to its average number $n_{p}\left(=1 /\left[\exp \left(E_{\mathrm{op}} / k T\right)-1\right]\right)$ or $n_{\mathrm{p}}+1$, respectively, where $E_{\mathrm{op}}=0.03 \mathrm{eV}$ is the energy of the optical phonon. Thus an electron is expected to lose a small amount of energy $E_{\mathrm{s}}=E_{\mathrm{op}}\left[\left(n_{p}+1 / 2 n_{p}+1\right)-\right.$
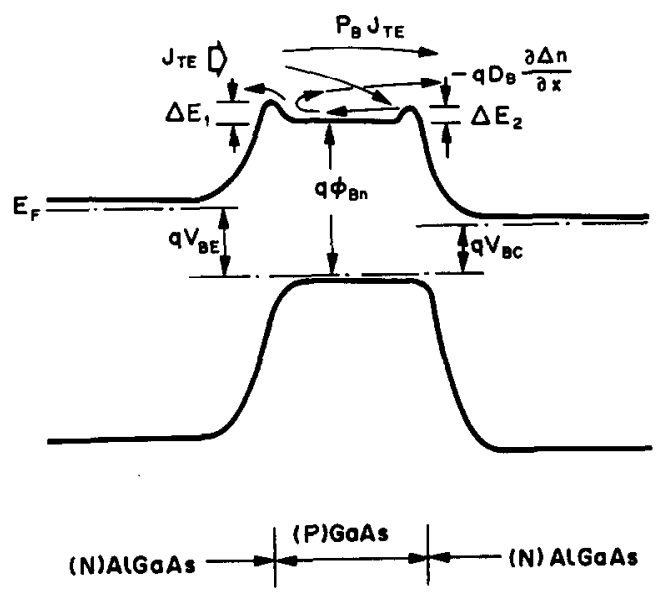

Fig. 1. Schematic band diagram showing the various current transport mechanisms across the base including the hot electron effect. 
$\left.\left(n_{p} / 2 n_{p}+1\right)\right] \sim 0.016 \mathrm{eV}$ during a scattcring event at room temperature. The energy of hole plasmon is $0.015 \mathrm{eV}$ in the base layer doped to $1 \times 10^{18} / \mathrm{cm}^{3}$, therefore, these two scattering mechanisms are treated to be the same one with mean free path $l$. The fraction $P_{\mathrm{B}}$ of those injected electrons which are "hot" is thus given by:

$$
P_{\mathrm{B}}=\sum_{n=0}^{m} P_{n}=\sum_{n=0}^{m} \frac{(W / l)^{n}}{n l} \exp \left(-\frac{W}{l}\right),
$$

where $W$ is the neutral base width and $P_{n}$ is the Poisson distribution[15] which represents the probability that an electron will undergo $n$ scattering events while transporting through base. $m$ is the maximum allowable number of scattering events defined by $\left(\Delta E_{1}-\Delta E_{2}\right) / E_{\mathrm{s}}$. The remainders are those electrons that suffer reflection by $\Delta E_{2}$ in the first passage and can be regarded as Boltzmann gas which goes on the standard diffusion process as described in the previous theory[8]. Therefore, in this simplified model, the forward-transferred $I-V$ characteristics, which neglect the recombination loss of hot electrons with holes in the base, is governed by the following boundary conditions:

(i) at $x=0$, the $e-b$ junction depletion layer edge at base side:

$$
\begin{aligned}
J_{\mathrm{E}} & =J_{\mathrm{TE}}-\frac{q v_{n} \Delta n(0)}{4} \exp \left(-\frac{\Delta E_{1}}{k T}\right) \\
& =-\left.q D_{\mathrm{B}} \frac{\partial \Delta n}{\partial x}\right|_{x=0}+P_{\mathrm{B}} J_{\mathrm{TE}}
\end{aligned}
$$

(ii) at $x=W$, the $b-c$ junction depletion layer edge at base side:

$$
\begin{aligned}
\mathrm{J}_{\mathrm{C}} & =P_{\mathrm{B}} J_{\mathrm{TE}}-\left.q D_{\mathrm{B}} \frac{\partial \Delta n}{\partial x}\right|_{x=W} \\
& =P_{\mathrm{B}} J_{\mathrm{TE}}+\frac{q v_{n} \Delta n(W)}{4} \exp \left(-\frac{\Delta E_{2}}{k T}\right),
\end{aligned}
$$

where $J_{\mathrm{TE}}$ is the thermionic emission current density over the $e-b$ junction potential spike and defined as:

$$
J_{\mathrm{TE}}=A^{*} T^{2} \exp \left(\frac{q \phi_{\mathrm{B} n}+\Delta E_{1}}{k T}\right) \exp \left(\frac{q V_{\mathrm{BE}}}{k T}\right),
$$

where $A^{*}$ is the Richarson constant employing the GaAs effective mass and $V_{\mathrm{BE}}$ is the applied $e-b$ junction bias. $q \phi_{B n}$ is the barrier height as defined in Fig. 1. $J_{\mathrm{E}}$ is the net injection electron current density across the $e-b$ junction and $J_{\mathrm{C}}$ is the transferred collector current density. Other parameters have their usual meaning[14]. The boundary conditions of the reverse-transferred characteristics are similar to those of forward-transferred characteristics. The final formula appear to be very simple, which is a combina- tion of two terms i.e.:

$$
\begin{aligned}
& J_{\mathrm{E}}=P_{\mathrm{B}} J_{\mathrm{TE}}+\left(1-P_{\mathrm{B}}\right) \frac{J_{\mathrm{TE}}}{R_{n 2}}, \\
& J_{\mathrm{C}}=P_{\mathrm{B}} J_{\mathrm{TE}}+\alpha_{2}\left(1-P_{\mathrm{B}}\right) \frac{J_{\mathrm{TE}}}{R_{n 2}},
\end{aligned}
$$

where $\alpha_{2}$ is the forward transport factor:

$$
\begin{aligned}
& \alpha_{2}=\frac{T_{2}}{T_{2} \cosh \left(\frac{W}{L_{\mathrm{B}}}\right)+\left(\frac{4 D_{\mathrm{B}}}{L_{\mathrm{B}} v_{n}}\right) \sinh \left(\frac{W}{L_{\mathrm{B}}}\right)}, \\
& T_{2}=\exp \left(-\frac{\Delta E_{2}}{k T}\right) .
\end{aligned}
$$

$L_{\mathrm{B}}$ and $v_{n}$ are the electron diffusion length and thermal velocity in the base, respectively. The second term in the denominator of $\alpha_{2}$ is the extra recombination loss due to bouncing phenomenon. $R_{n 2}[8]$ is a new parameter coming from the bouncing phenomenon of electrons between the $e-b$ and $b-c$ heterojunctions:

$$
\begin{aligned}
& R_{n 2}=1+T_{1} \frac{\left[\cosh \left(\frac{W}{L_{\mathrm{B}}}\right)+T_{2}\left(\frac{L_{B} v_{n}}{4 D_{\mathrm{B}}}\right) \sinh \left(\frac{W}{L_{\mathrm{B}}}\right)\right]}{\left[T_{2} \cosh \left(\frac{W}{L_{\mathrm{B}}}\right)+\left(\frac{4 D_{\mathrm{B}}}{L_{\mathrm{B}} v_{n}}\right) \sinh \left(\frac{W}{L_{\mathrm{B}}}\right)\right]}, \\
& T_{1}=\exp \left(-\frac{\Delta E_{1}}{k T}\right) .
\end{aligned}
$$

\section{EXPERIMENTS}

In order to study the hot electron effect, the $(N) \mathrm{Al}_{0.5} \mathrm{Ga}_{0.5} \mathrm{As}-(P) \mathrm{Al}_{0.23} \mathrm{Ga}_{0.77} \mathrm{As}-(p) \mathrm{GaAs}-(N) \mathrm{Al}_{0.15}$ $\mathrm{Ga}_{0.85}$ As DHBT's are grown on Si-doped (100) GaAs substrate by liquid-phase-epitaxy at about $800^{\circ} \mathrm{C}$. The fabrication processes are the same as Ref.[4]. The areas of $e-b$ and $b-c$ junctions are $1.33 \times 10^{-4}$ and $9.5 \times 10^{-4} \mathrm{~cm}^{2}$, respectively. In order to observe the hot electron effect, the thickness of $(p) \mathrm{GaAs}$ base is kept below $500 \AA$, as shown in Fig. 2(a). In addition, a $500 \AA$ thick $P$-type $\mathrm{Al}_{0.23} \mathrm{Ga}_{0.77}$ As is inserted at the $e-b$ junction interface to provide launching pad for injecting hot electrons[17]. Their doping concentrations are $1 \times 10^{18}$ and $1 \times 10^{16} / \mathrm{cm}^{3}$, respectively. The emitter and collector have the same doping concentration of $1 \times 10^{17} / \mathrm{cm}^{3}$. Since the sandwiched $P$-type $\mathrm{Al}_{0.23} \mathrm{Ga}_{0.77}$ As is completely depleted under normal forward bias due to its low doping and thin layer thickness, the potential spike $\Delta E_{1}$ that electron sees locates near the $P-p$ heterojunction interface as shown in Fig. 2(b).

Because of the effective mass mismatch at the $(N) \mathrm{Al}_{0.5} \mathrm{Ga}_{0.5} \mathrm{As} /(P) \mathrm{Al}_{0.23} \mathrm{Ga}_{0.77}$ As interface, the $X$-valley electrons of $(N) \mathrm{Al}_{0.5} \mathrm{Ga}_{0.5}$ As layer will suffer reflection and transfer to the $\Gamma$-valley of (P) $\mathrm{Al}_{0.23} \mathrm{Ga}_{0.37} \mathrm{As}$ when they undergo a real space transfer across the interface[18]. The similar thing happens when electrons transport across 


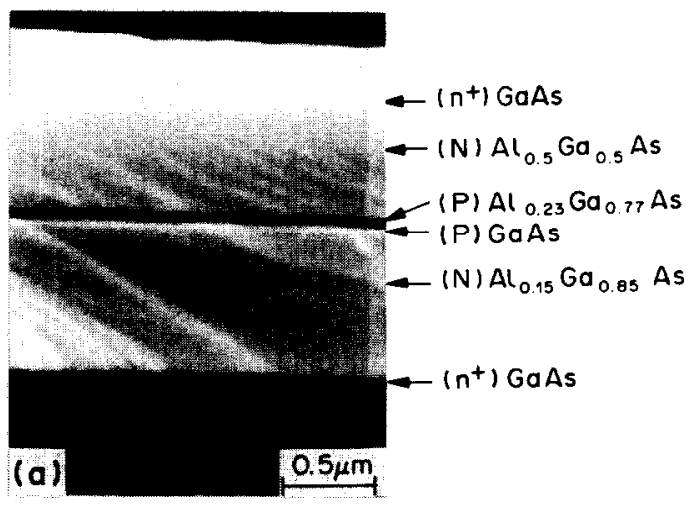

(b)

(P) $\mathrm{Al}_{0.23} \mathrm{Ga}_{0.77} \mathrm{As}$ (P) GaAs

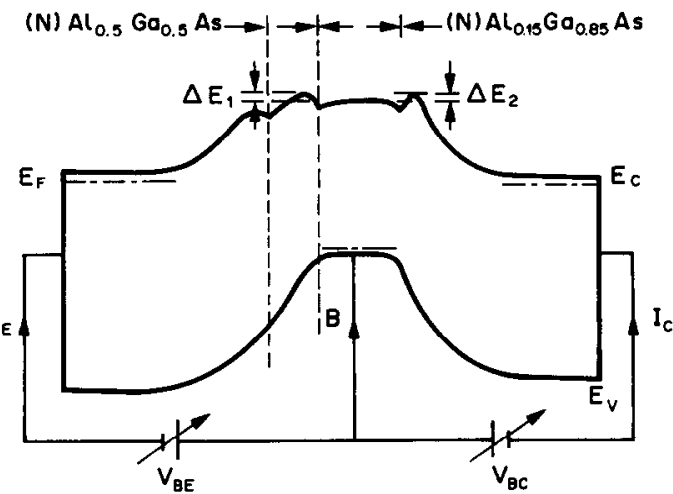

Fig. 2. (a) The scanning-electron-microscopy photograph of cross section and (b) the band diagram of the DHBT.

(P) $\mathrm{Al}_{0.23} \mathrm{Ga}_{0.77} \mathrm{As} /\left(P^{+}\right) \mathrm{GaAs}$ interface. These scattering events at the heterojunction interface, however, can be fully taken care of by using the effective mass of GaAs instead of AlGaAs in the Richardson's constant of the thermionic emission theory[18]. Therefore, the electron transport across the forwardbiased depletion region in the $P$-AlGaAs layer can be simply treated as the thermionic emission current and there is no need to consider any scattering event in this layer.

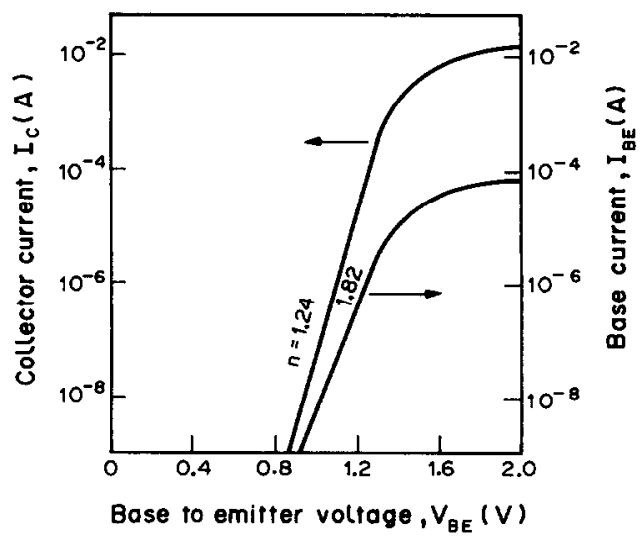

Fig. 3. The forward-transferred collector current $I_{\mathrm{C}}$ and base current $I_{\mathrm{BE}}$ vs the $e-b$ junction bias $V_{\mathrm{BE}}$ with the $b c$ junction short-circuited.

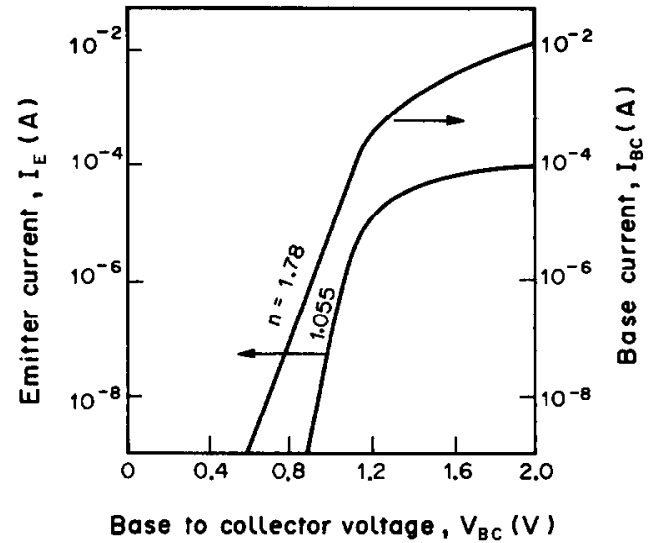

Fig. 4. The reverse-transferred emitter current $I_{\mathrm{E}}$ and base current $I_{\mathrm{BC}}$ vs the $b c$ junction bias $V_{\mathrm{BC}}$ with the $e-b$ junction short-circuited.

\section{RESULTS AND DISCUSSION}

Figures 3 and 4 show the forward and reversetransferred $I-V$ characteristics of DHBT from which the heterojunction potential spikes and $2 k T$ recombination current can be extracted as follows $[8,14,19]$ :

$$
\begin{aligned}
\Delta E_{1} & =0.195\left(V_{\mathrm{BE}}+0.01\right) \\
I_{\mathrm{BE}} & =3.13 \times 10^{-18} \exp \left(\frac{q V_{\mathrm{BE}}}{1.82 k T}\right) \\
\Delta E_{2} & =0.052\left(V_{\mathrm{BC}}+2.4\right) \\
I_{\mathrm{BC}} & =2.8 \times 10^{-15} \exp \left(\frac{q V_{\mathrm{BC}}}{1.78 k T}\right),
\end{aligned}
$$

where $I_{\mathrm{BE}}$ and $I_{\mathrm{BC}}$ represent the forward and reverse base currents, respectively, and are regarded as $2 k T$ recombination currents in the theoretical simulations. The value of 2.4 in the expression of $\Delta E_{2}$ was derived

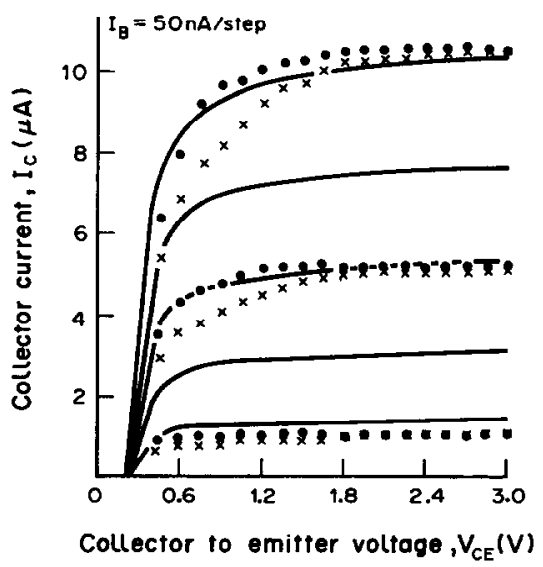

Fig. 5. The common-emitter $I-V$ characteristics of DHBT. The solid curves are the measured results. The crosses indicate the simulation results not including the hot electron effect and the dots are the results including hot electron effect with $58 \AA$ electron-phonon or electron-holc plasmon mean free path. 
by comparing the reverse-transferred emitter current $I_{\mathrm{E}}$, shown in Fig. 4, with that of single HBT with no $e-b$ junction spike[19]. The solid curves in Fig. 5 are the measured common-emitter $I-V$ characteristics of the test device. The base current is chosen in the submicro-ampere regime to avoid the series resistance effect. The crosses are the simulation result for $I_{\mathrm{B}}=$ 50,150 and $250 \mathrm{nA}$, respectively, using above parameters and a diffusion length of $7 \mu \mathrm{m}$ but not including the hot electron effect $[8,14]$. Clearly, a pronounced reach-through effect appears, for example, at $I_{\mathrm{C}} \sim 6 \mu \mathrm{A}$ in the saturation region of the curve for $I_{\mathrm{B}}=250 \mathrm{nA}$. This characteristic is certainly different from that of the experimental result. The physical reason underlying the reach-through effect has been explained in details in Ref.[14]. After including the hot electron effect with a $58 \AA$ electron-phonon or electron-hole plasmon mean free path, the simulation result can fit the measured curve very well. This mean free path value is exactly the same as in Ref.[20]. It implies that the carrier confinement by $b-c$ junction potential spike is reduced at least for $I_{\mathrm{C}}>6 \mu \mathrm{A}$ for the curve of $I_{\mathrm{B}}=250 \mathrm{nA}$ and the transport mechanism of hot electrons plays a significant role in the transistor $I-V$ characteristics.

\section{CONCLUSIONS}

In conclusion, the transport mechanism of nonequilibrium electrons injected over the heterojunction is incorporated into the theoretical model. The transport of hot electrons can play a significant role in the $I-V$ characteristics and can be probed through the curve fitting of common-cmitter $I-V$ characteristics. The pronounced reach-through effect will be reduced in a DHBT with short base and adequate $\Delta E_{2}$ by hot electron effect.
Acknowledgement-This work is supported by National Science Council of Republic of China under contract No. NSC-77-0404-E-002-12.

\section{REFERENCES}

1. H. Beneking and L. M. Su, Electron. Lett. 18, 25 (1982).

2. C. Dubon, R. Azoulay, P. Desrousseaux, J. Dangla, A. M. Duchenois, M. Houlntondji and D. Ankri, Int. Electron Dev. Meeting. Tech. Dig. p. 689 (1983).

3. J. R. Hayes, F. Capasso, R. J. Malik, A. C. Gossard and W. Wiegmann, Int. Electron Dev. Meeting. Tech. Dig. p. $686(1983)$.

4. S. C. Lee, J. N. Kau and H. H. Lin, J. appl. Phys. 58, $890(1985)$

5. S. L. Su, O. Tejayadi, T. J. Drummond, R. Fischer and H. Morkoc, IEEE. Electron Det. Lett. EDL-4, 130 (1983).

6. J. R. Hayes, F. Capasso, R. J. Malik, A. C. Gossard and W. Wiegmann, Electron. Lett. 19, 410 (1983).

7. N. C. Russ, R. Fischer and H. Morkoc, Appl. Phys. Lett. 47, 313 (1985).

8. S. C. Lee and H. H. Lin, J. appl. Phys. 59, 1688 (1986).

9. H. Kromer, Proc. IEEE 70, 13 (1982).

10. H. Kromer, J. Vac. Sci. Technol. B2, 126 (1983).

11. K. Tomizawa, Y. Awano and N. Hashizume, IEEE. Electron Dev. Lett. EDL-5, 362 (1984).

12. C. M. Maziar, M. E. Klausmeier-Brown, S. Bandyopadhyay and M. S. Lundstrom, IEEE Trans. Electron Dev. ED-33, 881 (1986).

13. J. R. Hayes, 44th Device Research Conference, University of Massachusetts (1986).

14. C. Z. Chen and S. C. Lee, IEEE Trans. Electron Dev. ED-34, 1463 (1987).

15. P. G. Hoel, S. C. Port and C. J. Stone, Introduction to Probability Theory, Chap. 9. Houghton Mifflin Company, Boston (1980).

16. A. F. J. Levi, J. R. Hayes, A. C. Gossard and J. H. English, Appl. Phys. Lett. 50, 98 (1987).

17. C. Z. Chen, S. C. Lee and H. H. Lin, J. appl. Phys. 62, 3976 (1987).

18. C. R. Crowell, Solid-St. Electron. 8, 395 (1965).

19. H. H. Lin and S. C. Lee, IEEE Electron Dev. Lett. EDL-6, 431 (1985).

20. S. M. Sze, Physics of Semiconductors Devices, 2 nd edn, p. 851. Wiley, New York (1981). 